\title{
The effect of vascular risk factor burden on the severity of COVID-19 illness, a retrospective cohort study
}

Houwei Du ${ }^{1,2^{*}+} \mathbb{D}$, Xiaobin Pan $^{3+}$, Nan Liu ${ }^{1,4+}$, Junnian Chen ${ }^{5}$, Xiaoling Chen ${ }^{6}$, David J. Werring ${ }^{7}$, Gareth Ambler ${ }^{8}$, Xiaoqing Li ${ }^{9}$, Ronghua Chen ${ }^{1,2}$, Yixian Zhang ${ }^{4}$, Huayao Huang ${ }^{4}$, Feifei Lin ${ }^{1}$, Pincang Xia ${ }^{9}$, Chao Chen ${ }^{10}$, Zhenyang Zheng ${ }^{1,2}$, Sangru Wu ${ }^{1}$, Hanhan Lei ${ }^{1}$, Lei Gao ${ }^{11}$, Mingxu Huang ${ }^{12}$, Kexu Lin ${ }^{12}$, Xiaoping X ${ }^{13}{ }^{3}$, Yukun Luo ${ }^{14}$, Ziwen Zhao ${ }^{14}$, Chen Li ${ }^{15}$, Hailong Lin ${ }^{16}$, Yu Lin ${ }^{17}$, Zhenghui Huang ${ }^{18}$, Rongxiang Cao ${ }^{18}$, Limin Chen ${ }^{18}$ and On behalf of the Fujian Medical Team Support Wuhan for COVID-19

\begin{abstract}
Background: Patients with cardiovascular comorbidities are at high risk of poor outcome from COVID-19. However, how the burden (number) of vascular risk factors influences the risk of severe COVID-19 disease remains unresolved. Our aim was to investigate the association of severe COVID-19 illness with vascular risk factor burden.

Methods: We included 164 (61.8 \pm 13.6 years) patients with COVID-19 in this retrospective study. We compared the difference in clinical characteristics, laboratory findings and chest computed tomography (CT) findings between patients with severe and non-severe COVID-19 illness. We evaluated the association between the number of vascular risk factors and the development of severe COVID-19 disease, using a Cox regression model.

Results: Sixteen (9.8\%) patients had no vascular risk factors; 38 (23.2\%) had 1; 58 (35.4\%) had 2; 34 (20.7\%) had 3; and $18(10.9 \%)$ had $\geq 4$ risk factors. Twenty-nine patients (17.7\%) experienced severe COVID-19 disease with a median (14 [7-27] days) duration between onset to developing severe COVID-19 disease, an event rate of 4.47 per 1000-patient days (95\%Cl 3.10-6.43). Kaplan-Meier curves showed a gradual increase in the risk of severe COVID-19 illness (log-rank $P<0.001$ ) stratified by the number of vascular risk factors. After adjustment for age, sex, and comorbidities as potential confounders, vascular risk factor burden remained associated with an increasing risk of severe COVID-19 illness.

Conclusions: Patients with increasing vascular risk factor burden have an increasing risk of severe COVID-19 disease, and this population might benefit from specific COVID-19 prevention (e.g., self-isolation) and early hospital treatment measures.
\end{abstract}

Keywords: Vascular risk factor, Coronavirus disease 2019, Prognosis

\footnotetext{
*Correspondence: duhouwei@outlook.com; houweidu@fjmu.edu.cn

${ }^{\dagger}$ Houwei Du, Xiaobin Pan and Nan Liu contributed equally to this work.

'Stroke Research Center, Department of Neurology, Fujian Medical University

Union Hospital, 29 Xinquan Road, Gulou District, Fuzhou 350001, China

${ }^{2}$ Institute of Clinical Neurology, Fujian Medical University, Fuzhou, China

Full list of author information is available at the end of the article
}

(c) The Author(s). 2020 Open Access This article is licensed under a Creative Commons Attribution 4.0 International License, which permits use, sharing, adaptation, distribution and reproduction in any medium or format, as long as you give appropriate credit to the original author(s) and the source, provide a link to the Creative Commons licence, and indicate if changes were made. The images or other third party material in this article are included in the article's Creative Commons licence, unless indicated otherwise in a credit line to the material. If material is not included in the article's Creative Commons licence and your intended use is not permitted by statutory regulation or exceeds the permitted use, you will need to obtain permission directly from the copyright holder. To view a copy of this licence, visit http://creativecommons.org/licenses/by/4.0/ The Creative Commons Public Domain Dedication waiver (http://creativecommons.org/publicdomain/zero/1.0/) applies to the data made available in this article, unless otherwise stated in a credit line to the data. 


\section{Background}

The coronavirus disease 2019 (COVID-19) is a highly infectious viral respiratory illness caused by severe acute respiratory syndrome coronavirus 2 (SARS-CoV-2), which was first reported in December 2019 in Wuhan, China [1]. COVID-19 rapidly became a worldwide pandemic, affecting more than 28 million people [2]. Cardiovascular risk factors, including ischemic heart disease, hypertension and diabetes, are common in patients with COVID-19 infection, and associated with mortality $[3,4]$. Cardiovascular diseases are a major public health challenge worldwide. For example, a population-based study with a large sample size showed that $6.63 \%(3070 / 46,285)$ of people had at least one major cardiovascular disease [5]. A global cohort study showed that 57,303 (39.4\%) people had hypertension, 15,900 (10.2\%) had diabetes and 26,691 (18.5\%) reported low physical activity, and that mean body-mass index (BMI) was 25.7 (SD 5.3) $\mathrm{kg} / \mathrm{m}^{2}$ [6]. Given the vast number of people living with prevalent vascular risk factors and the rapid global spread of the COVID-19 pandemic, it is essential to know how vascular risk factors influence the risk of developing severe COVID-19 disease. Most previous studies showed an association between vascular risk factors (such as hypertension and diabetes) and COVID-19 severity [7]. However, little is known about how patients stratified by burden of vascular risk factors differ from each other, and whether the number of vascular risk factors is associated with COVID-19 disease severity. We hypothesized that in patients infected with SARSCoV-2 increasing vascular risk factor burden increases the risk of developing severe illness. We therefore investigated the association between the number of vascular risk factors and severe COVID-19 disease in this observational retrospective study.

\section{Methods}

\section{Study design and participants}

This is a single-center, retrospective, observational study done at Tumor Center of Union Hospital, Tongji Medical College, Huazhong University of Science and Technology (Wuhan, China), which is a designated hospital to treat patients with COVID-19 disease by national medical teams to support Wuhan. We analyzed admitted patients between 15 February and 14 March 2020, who had been diagnosed with COVID-19 disease based on World Health Organization interim guidance [8]. Laboratory confirmation of COVID-19 infection was performed by the local health authority as previously described [9].

\section{Data collection and outcome measures}

We retrospectively reviewed the electronic medical records of 164 consecutive eligible patients with COVID19 using a digital database. Two physicians (J.C and X.C) extracted the epidemiological, demographic, clinical, laboratory data on admission, and treatment data using a standardized data collection form. Data on the most intense level of oxygen treatment during hospitalization (nasal cannula, medical mask, high solution and invasive mechanical ventilation) was also collected. Two physicians (K.L and X.P) collected the outcome data, which was adjudicated by two senior physicians (H.D. and Y.L.) blinded to baseline demographics. Two physicians (G.L and R.C) reviewed the chest $\mathrm{CT}$ images blindly. In cases of disagreement, a consensus was reached after discussion with a senior respiratory physician (L.C.) and a radiologist (H.L). If data were missing or uncertain from the medical records, we obtained and clarified data by direct communication with attending doctors and other healthcare providers.

Definitions of vascular risk factors were based on previous literature $[6,10-13]$ as follows. Hypertension was defined as documented systolic blood pressure $\geq 140$ $\mathrm{mmHg}$ and/or diastolic blood pressure $\geq 90 \mathrm{mmHg}$, or by patients' self-reported diagnosis of hypertension and/ or by the treatment of antihypertensives [10, 11]. Diabetes was defined as fasting blood glucose $\geq 7.0 \mathrm{mmol} / \mathrm{L}$ and/or a previous diagnosis of diabetes and/or by the treatment of antidiabetic drugs [10]. Dyslipidaemia was defined as $\mathrm{TC} \geq 6.22 \mathrm{mmol} / \mathrm{L}$ and/or $\mathrm{TG} \geq 2.26 \mathrm{mmol} / \mathrm{L}$ and/or $\mathrm{HDL}-\mathrm{C}<1.04 \mathrm{mmol} / \mathrm{L}$ and $/$ or $\quad$ LDL-C $\geq 4.14$ $\mathrm{mmol} / \mathrm{L}$ [10]. We classified patients as having atrial fibrillation (AF) if they had been diagnosed with AF by electrocardiogram at admission or during hospital stay [11]. Current smoking ( $\geq 1$ cigarette per day) and regular drinker (any dose of alcohol, $\geq 1$ time per week) were defined by patients' self-report [12]. Physical inactivity was defined as less than 150 min per week of moderate activity or less than 75 min per week of vigorous activity [13], and overweight as body mass index (BMI) $\geq 25 \mathrm{~kg} / \mathrm{m}^{2}$ [10]. We counted and aggregated the following vascular risk factors to construct a vascular risk factor score in each patient: hypertension, diabetes, dyslipidemia, atrial fibrillation, current smoker, overweight and physical inactivity. These factors are considered well-documented risk factors for cardiovascular diseases, including cerebrovascular disease [6, 10-14]. Our primary outcome was severe COVID-19 disease defined as fever or suspected respiratory infection, plus one of: respiratory rate $>30$ breaths $/ \mathrm{min}$; severe respiratory distress; or $\mathrm{SPO}_{2} \leq 93 \%$ on room air based on the interim guidance of the World Health Organization [8]. Our secondary outcome was death, which was limited by the duration of our observation period. All the authors agreed on the study protocol and reviewed the manuscript.

\section{Statistical analysis}

Continuous data were summarized using means with standard deviations or medians with interquartile ranges 
(IQR), and categorical data were summarized as counts with percentages. We used the t-test or Mann-Whitney test to compare differences in continuous variables, and the chi-square test or Fisher's exact test to compare differences in categorical variables. We calculated the absolute event rate per 1000 patient-days for severe COVID19 illness and death during our observation period. We also compared event rates during our observation period stratified by the number of vascular risk factors (none, 1 , 2, 3, or $\geq 4$ risk factors). Kaplan-Meier curves depicted the risk for outcome events stratified by the number of vascular risk factors (none, 1, 2, 3, or $\geq 4$ risk factors). We used univariable Cox regression analysis to evaluate the association between vascular risk factor burden and severe COVID-19 pneumonia. For multivariable analysis, we chose variables based on previous findings and clinical constraints to avoid overfitting the Cox regression model [15]. We therefore included chronic obstructive pulmonary disease (COPD), cardio-cerebrovascular disease, tumor, renal impairment, decreased leucocytes, decreased lymphocytes, increased lactate dehydrogenase (LDH) and chest CT findings separately as potential confounders, in addition to age and sex in the regression model. We also performed sensitivity analyses using the E-value approach, where the E-value is defined as the minimum strength of association on the risk ratio scale that an unmeasured confounder would need to have with both the exposure and the outcome, conditional on the measured covariates, to fully explain away a specific exposure-outcome association [16]. We calculated the E-value using an online E-value calculator (https:// mmathur.shinyapps.io/evalue/). All analyses were performed using STATA 12.0 (StataCorp LP, College Station, TX) and SPSS for Windows (SPSS 25.0, IBM, Inc., Chicago, IL, USA).

\section{Results}

In this analysis, we included 164 consecutive patients (61.8 \pm 13.6 years) who had COVID-19 disease between 15 February and 14 March 2020. Sixteen (9.8\%) patients had no vascular risk factor, whereas $38(23.2 \%)$ had 1,58 (35.4\%) had 2, 34 (20.7\%) had 3, and 18 (10.9\%) had $\geq 4$ risk factors. The demographics, clinical and radiological characteristics in patients with severe COVID-19 illness and non-severe COVID-19 illness are shown in Table 1. Patients with severe COVID-19 disease were similar to those with non-severe illness regarding their previous history of diabetes, COPD, tumor, treatment with immunosuppressives, exposure to wet seafood market, and onset symptoms. Patients with severe disease were older $(71.0 \pm 12.8$ vs $59.8 \pm 12.9, p<0.001)$, more likely to be male (20 [69.0\%] vs 64 [47.4\%], $p=0.04)$, and more likely to have pre-existent cardio-cerebrovascular disease $(8$ [27.6\%] vs 15 [11.1\%], $p=0.02)$. Regarding vascular risk factors, patients with severe COVID-19 disease were more likely to have hypertension (13 [44.8\%] vs 39 [28.9\%], $p=0.09)$, dyslipidemia (18 [62.1\%] vs 45 [33.3\%], $p=0.004)$, overweight (14 [48.3\%] vs 41 [30.4\%], $p=0.064)$ and physical inactivity $(26[89.7 \%]$ vs 77 [57.0\%], $p=0.001$ ). The severe COVID-19 event rate increased with the number of vascular risk factors (Table 2). Looking at routine blood test findings, patients with severe COVID-19 disease were more likely to have decreased leucocytes $(17.2 \%$ vs $4.4 \%, p=0.01)$, decreased lymphocytes $(65.5 \%$ vs $26.7 \%, p<0.001)$, and increased lactic dehydrogenase (LDH) $(58.6 \%$ vs $24.6 \%$, $\mathrm{p}<0.001)$. Regarding chest computed tomography $(\mathrm{CT})$ findings, patients with severe COVID-19 illness were more likely to be bilaterally affected.

The median follow-up time was 42 days [IQR 35-49], providing 6493 patient-days of data. One hundred and three $(62.8 \%)$ patients were cured at discharge at the end of our observation period. Twenty-nine patients (17.7\%) experienced severe COVID-19 illness with a median (14 [7-27] days) duration between symptom onset to developing a severe disease, an event rate of 4.47 per 1000patient days (95\%CI 3.10-6.43). Six patients (3.7\%) died during hospitalization, an event rate of 0.84 per 1000 patient days (95\%CI 0.38-1.88). Kaplan-Meier curves showed that the risk of severe COVID-19 illness tended to increase with the number of vascular risk factors (log rank $P<0.001$, Fig. 1). In univariable analysis, the risk of severe COVID-19 increased with increasing vascular risk factor burden (unadjusted HR 2.06, 95\% CI 1.51-2.80). After adjustment for age and sex, the vascular risk factor burden remained significantly associated with severe COVID-19. (adjusted OR 1.55 95\%CI 1.09-2.21, Table 3). This relationship did not change after additional confounder adjustment for, separately, COPD, cardiocerebrovascular disease, tumor and renal impairment, in addition to age and sex (Table S1). Separate adjustment for routine blood examinations and chest CT findings, in addition to age and sex, did not alter our results (Table S2). Sensitivity analyses revealed that it would need moderately robust unobserved confounding to render the exposure-outcome association null. (Table 3, Table S1 and Table S2). For example, an E-value of 2.12 for the estimate (Table 3, and Figure S1) indicates that the observed risk ratio of 2.12 could only be explained away by an unmeasured confounder that was associated with both the exposure and the outcome by risk ratios of more than two, above and beyond the measured confounders, but weaker confounding could not do so [16].

\section{Discussion}

Our most important finding is that in COVID-19 patients increasing vascular risk factor burden is associated with an increasing risk of experiencing severe COVID- 
Table 1 Demographic, clinical, laboratory, radiological characteristics, treatment and outcome between severe and non-severe COVID-19 pneumonia

\begin{tabular}{|c|c|c|c|c|}
\hline & Total $(n=164)$ & Severe $(n=29)$ & Non-severe $(n=135)$ & $P$ \\
\hline Age, $(y)$ mean $\pm S D$ & $61.8 \pm 13.6$ & $71.0 \pm 12.8$ & $59.8 \pm 12.9$ & $<0.001$ \\
\hline Male, n (\%) & $84(51.2)$ & $20(69.0)$ & $64(47.4)$ & 0.04 \\
\hline Current smoker, n (\%) & $17(10.4)$ & $2(6.9)$ & $15(11.1)$ & 0.73 \\
\hline Regular drinker, n (\%) & $3(1.8)$ & 0 & $3(2.2)$ & $>0.99$ \\
\hline Hypertension, n (\%) & $52(31.7)$ & $13(44.8)$ & $39(28.9)$ & 0.09 \\
\hline Diabetes, n (\%) & $31(18.9)$ & $7(24.1)$ & $24(17.8)$ & 0.43 \\
\hline Dyslipidemia, n (\%) & $63(38.4)$ & $18(62.1)$ & $45(33.3)$ & 0.004 \\
\hline Atrial fibrillation, n (\%) & $10(6.1)$ & $4(13.8 \%)$ & $6(4.4 \%)$ & 0.14 \\
\hline Overweight, n (\%) & $55(33.5)$ & $14(48.3)$ & $41(30.4)$ & 0.06 \\
\hline Physical inactivity, n (\%) & $103(62.8)$ & $26(89.7)$ & $77(57.0)$ & 0.001 \\
\hline COPD, n (\%) & $12(7.3)$ & $4(13.8)$ & $8(5.9)$ & 0.28 \\
\hline Cardio-cerebrovascular disease, n (\%) & $23(14.0)$ & $8(27.6)$ & $15(11.1)$ & 0.020 \\
\hline Renal impairment, n (\%) & $25(15.2)$ & $10(34.5)$ & $15(11.1)$ & 0.001 \\
\hline Digestive disease, n (\%) & $15(9.1)$ & $1(3.4)$ & $14(10.4)$ & 0.41 \\
\hline Immunosuppresives, n (\%) & $3(1.8)$ & $1(3.4)$ & $2(1.5)$ & $>0.99$ \\
\hline Tumor, n (\%) & $13(7.9)$ & $3(10.3)$ & $10(7.4)$ & 0.88 \\
\hline Wet market exposure, n (\%) & $2(1.2)$ & $1(3.4)$ & $1(0.7)$ & 0.79 \\
\hline \multicolumn{5}{|l|}{ Clinical symptoms } \\
\hline Fever, n (\%) & $115(70.1)$ & $20(69.0)$ & $95(70.4)$ & 0.88 \\
\hline Dry cough, n (\%) & $104(63.4)$ & $19(65.5)$ & $85(63.0)$ & 0.80 \\
\hline Productive cough, n (\%) & $23(14.0)$ & $2(6.9)$ & $21(15.6)$ & 0.36 \\
\hline Fatigue, n (\%) & $57(34.8)$ & $9(31.0)$ & 48 (35.6) & 0.64 \\
\hline Muscle or joint ache, $\mathrm{n}(\%)$ & $21(12.8)$ & $2(6.9)$ & $19(14.1)$ & 0.46 \\
\hline Thoracalgia, n (\%) & $31(18.9)$ & $6(20.7)$ & $25(18.5)$ & 0.79 \\
\hline Sore throat, n (\%) & $23(14.0)$ & $4(13.8)$ & $19(14.1)$ & $>0.99$ \\
\hline Diarrhea, n (\%) & $13(7.9)$ & $4(13.8)$ & $9(6.7)$ & 0.36 \\
\hline Catarrh, n (\%) & $6(3.7)$ & 0 & $6(4.4)$ & 0.59 \\
\hline Anorexia, n (\%) & $48(29.3)$ & $8(27.6)$ & $40(29.6)$ & 0.83 \\
\hline Short of breath, n (\%) & 65 (39.6) & $15(51.7)$ & $50(37.0)$ & 0.14 \\
\hline Headache, n (\%) & 19 (11.6) & $3(10.3)$ & $16(11.9)$ & $>0.99$ \\
\hline Total symptoms (IQR) & $3[2-4]$ & $3[2-4]$ & $3[2-4]$ & 0.94 \\
\hline \multicolumn{5}{|l|}{ Routine blood examinations } \\
\hline Decreased leucocytes, n (\%) & $11(6.7)$ & $5(17.2)$ & $6(4.4)$ & 0.01 \\
\hline Decreased lymphocytes, n (\%) & $55(33.5)$ & $19(65.5)$ & $36(26.7)$ & $<0.001$ \\
\hline Decreased hemoglobin, n (\%) & $42(25.6)$ & $15(51.7)$ & $27(20.0)$ & $<0.001$ \\
\hline Decreased platelets, n (\%) & $14(8.5)$ & $7(24.1)$ & $7(5.2)$ & 0.001 \\
\hline Increased ALT or AST, n (\%) & $58(35.4)$ & $14(18.3)$ & $44(32.6)$ & 0.11 \\
\hline Increased LDH, n (\%) & $50(30.7)$ & $17(58.6)$ & $33(24.6)$ & $<0.001$ \\
\hline \multicolumn{5}{|l|}{ Complications } \\
\hline Acute stroke, n (\%) & $3(1.8)$ & $3(10.3)$ & 0 & 0.005 \\
\hline Shock, n (\%) & $3(1.8)$ & $3(10.3)$ & 0 & 0.005 \\
\hline CT findings, n (\%) & & & & 0.04 \\
\hline Unilateral pneumonia, n (\%) & $26(15.9)$ & $3(10.3)$ & $23(17.0)$ & \\
\hline
\end{tabular}


Table 1 Demographic, clinical, laboratory, radiological characteristics, treatment and outcome between severe and non-severe COVID-19 pneumonia (Continued)

\begin{tabular}{|c|c|c|c|c|}
\hline & Total $(n=164)$ & Severe $(n=29)$ & Non-severe $(n=135)$ & $P$ \\
\hline Bilateral pneumonia, n (\%) & $86(52.4)$ & $11(37.9)$ & $75(55.6)$ & \\
\hline Multiple mottling and ground-glass opacity, n (\%) & $52(31.7)$ & $15(51.7)$ & $37(27.4)$ & \\
\hline \multicolumn{5}{|l|}{ Treatment } \\
\hline Oxygen therapy, n (\%) & & & & $<0.001$ \\
\hline Nasal cannula, n (\%) & $79(48.2)$ & $16(55.2)$ & $63(46.7)$ & \\
\hline Medical mask, n (\%) & $5(3.0)$ & $5(17.2)$ & 0 & \\
\hline High solution, n (\%) & $5(3.0)$ & $5(17.2)$ & 0 & \\
\hline Invasive ventilation, n (\%) & $3(1.8)$ & $3(10.3)$ & 0 & \\
\hline Glucocorticoid, n (\%) & $20(12.2)$ & $9(31.0)$ & $11(8.1)$ & 0.001 \\
\hline Antibacterial, n (\%) & $117(71.8)$ & $23(82.1)$ & $94(69.6)$ & 0.18 \\
\hline Antivirus, n (\%) & $158(96.3)$ & $27(93.1)$ & $131(97.0)$ & 0.63 \\
\hline Chinese traditional medicine, $\mathrm{n}(\%)$ & $156(95.1)$ & $26(89.7)$ & $130(96.3)$ & 0.30 \\
\hline Outcomes Cured at discharge, n (\%) & $103(62.8)$ & $9(31.0)$ & $94(69.6)$ & $<0.001$ \\
\hline Death, n (\%) & $6(3.7)$ & $6(20.7)$ & 0 & $<0.001$ \\
\hline
\end{tabular}

Tumor was defined by patients' self-report of having a history of a malignant tumor. Decreased means below the lower limit of the normal range and increased means over the upper limit of the normal range

Abbreviations: COVID-19 coronavirus disease 2019, SD Standard deviation, COPD Chronic obstructive pulmonary disease, IQR Interquartile range, ALT Alanine transaminase (U/L; normal range 0-40), AST Alanine aminotransferase (U/L; normal range 0-40), LDH Lactate dehydrogenase (U/L; normal range 109-245, data available in 163 patients), CT Computed tomography, Leucocytes $\left(\times 10^{9} / \mathrm{L}\right.$; normal range $\left.3.5-9.5\right)$, Lymphocytes $\left(\times 10^{9} / \mathrm{L} ;\right.$ normal range $\left.1.1-3.2\right)$, Platelets $\left(\times 10^{9} / \mathrm{L}\right.$; normal range 125.0-350.0), Hemoglobin (g/L; normal range 130.0-175.0)

19 illness. The seven included vascular risk factors are generally readily available at hospital admission, which may help clinicians to identify patients with poor prognosis at an early stage.

Previous studies suggested patients with mild COVID19 disease were at low risk for an unfavorable outcome than those with severe COVID-19 disease $[4,15]$. Therefore, identifying risk factors for severe COVID-19 illness is essential to ensure timely management. In our cohort, symptoms at COVID-19 onset were not significantly different between patients with and without severe COVID-19 illness, suggesting that onset symptoms were not of prognostic relevance. The association between

Table 2 Number of cardiovascular risk factors and severe COVID-19 events

\begin{tabular}{ll}
\hline Vascular risk factor number & Severe COVID-19 event, $\mathbf{n}(\%)^{*}$ \\
\hline No risk factor $n=16$ & $1(6.3)$ \\
One risk factor $n=38$ & $2(5.3)$ \\
Two risk factors $n=58$ & $8(13.8)$ \\
Three risk factors $n=34$ & $8(23.5)$ \\
Four risk factors $n=15$ & $8(53.3)$ \\
Five risk factors $n=3$ & $2(66.7)$
\end{tabular}

Vascular risk factors include hypertension, diabetes, dyslipidemia, atrial

fibrillation, current smoker, overweight and physical inactivity

Abbreviations: COVID-19 coronavirus disease 2019

${ }^{*} P<0.001$ (chi-square test) total baseline vascular risk factor burden and severe COVID-19 illness in our cohort may inform the development of future risk stratification models.

Our data showed that patients with severe COVID-19 illness were more likely to have a baseline ischemic cardiac or cerebrovascular disease $(8[27.6 \%]$ vs 15 [11.1\%]). This result is line with findings of previous descriptive studies [3, 17]. Previous studies also showed individual vascular risk factors such as hypertension and diabetes were more frequent in severe COVID-19 patients [3, 14]. Moreover, data from Italy and the United States confirmed increased mortality rates in patients with comorbidities, particularly the older population with pre-existent cardiovascular conditions $[18,19]$. Our findings showed vascular risk factors were prevalent among COVID-19 patients. Only sixteen (9.8\%) patients had no vascular risk factor, whereas twothirds of patients had more than one risk factor. Patients with increasing vascular risk factor burden in our cohort were older $(p<0.001)$, having a high risk for the severe COVID-19 disease, and consequently underwent more aggressive ventilation. To the best of our knowledge, the association between total vascular risk factor burden and the severity of COVID-19 has not been systematically investigated before. Our data therefore expands upon previous findings of individual comorbidities as risk factors for severe COVID-19 disease and may be relevant for important public health and clinical decisions regarding the management of the current COVID-19 and future pandemics. 


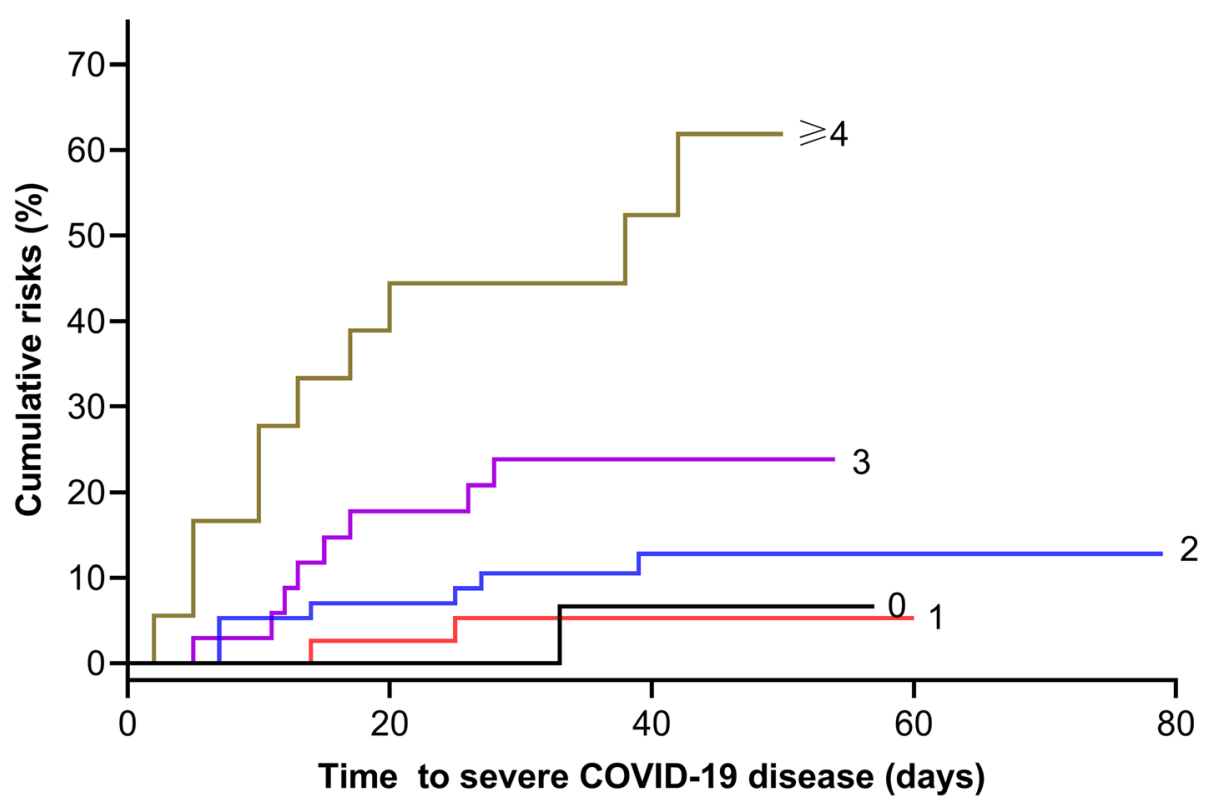

Fig. 1 Cumulative probability of severe COVID-19 disease stratified by the number of vascular risk factors. Figure legend: COVID-19=coronavirus disease 2019

Among the vascular risk factors associated with severe COVID-19, being overweight showed a strong association [20]. People with obesity around the world are at high risk for severe COVID-19 illness [21]. For example, a previous study has shown that overweight patients had a 1.84-fold higher risk of developing severe COVID-19 (95\% CI 0.99-3.43) than normal-weight patients [22]. Moreover, our data indicate that patients with physical inactivity are more likely to have severe COVID-19 illness. The relationship between these lifestyle factors and COVID-19 severity warrants further studies for clarification, but in the meantime our findings further strengthen the idea that the population at risk of COVID-19 should keep exercising at home during the quarantine. Moreover, considering the strict isolation strategies to curb virus spreading, the effect of physical inactivity on the potential risk of the cardiocerebrovascular events needs further urgent investigation worldwide.
Whether some antihypertensives could increase the susceptibility to developing severe forms of COVID19 remains uncertain. SARS-CoV-2 is linked to the renin-angiotensin system (RAS), which plays an essential role in regulating blood pressure and the pathophysiology of cardiovascular disease [23]. Patients with pre-existing vascular risk factor burden such as hypertension and diabetes were likely to be treated with angiotensin-converting enzyme inhibitors (ACEI) or angiotensin receptor blockers (ARB). Both ACEI and ARB can upregulate ACE2, facilitating the SARSCoV-2 entry into pneumocytes, and might cause exacerbation of the underlying disease [24]. However, available clinical evidence does not confirm an association between the use of ACEI or ARB and the poor outcomes of COVID-19 [7, 25, 26]. Long-term studies in larger numbers of hospitalized COVID-19 patients receiving ACEI and ARB therapy are needed to address this question.

Table 3 Association between vascular risk factor burden and severe COVID-19 illness and sensitivity analyses using the E-value approach

\begin{tabular}{|c|c|c|c|c|}
\hline & Unadjusted & & Age- and sex- adjusted & \\
\hline E-value & NA & & 2.12 & \\
\hline \multirow[t]{2}{*}{ Number of vascular risk factor } & $\mathrm{HR}(95 \% \mathrm{Cl})$ & $p$ value & $\mathrm{HR}(95 \% \mathrm{Cl})$ & $p$ value \\
\hline & $2.06[1.51-2.80]$ & $<0.001$ & $1.55[1.09-2.21]$ & 0.01 \\
\hline
\end{tabular}

Abbreviations: COVID-19 coronavirus disease 2019, HR hazard ratio

Vascular risk factors include hypertension, diabetes, dyslipidemia, atrial fibrillation, current smoker, overweight and physical inactivity

E-values represent the minimum strength of association an unmeasured confounder would have to possess between both the exposure and the outcome in

order to reduce the observed association between the exposure and outcome to 1 (no association) on the relative scale 
The underlying mechanisms of the association between vascular risk factor burden and COVID-19 severity remain unclear. Immobilization and high body mass were found to be risk factors for pulmonary embolism $[27,28]$, which were prevalent in severe COVID-19 disease $[29,30]$. Other possible explanations might include exaggerated systemic inflammation or a "cytokine storm". For example, metabolic risk factors such as obesity may increase the inflammation of the lung parenchyma and compromise the pulmonary function [31,32].

Our findings suggest that clinicians should pay close attention to the warning signs and symptoms of cardiac or cerebrovascular events in COVID-19 patients with high vascular risk factor burden. Establishing a multidisciplinary medical team of cardiologists, neurologists, respiratory specialists, and infectious disease specialists to offer rapid evaluation and acute treatment in COVID19 is urgently needed.

We acknowledge limitations. First, this is a retrospective study conducted at a single-centered hospital with limited sample size. Our findings need to be validated in further large sample-sized studies. Second, some vascular risk factors were self-reported, so their prevalence may be underestimated. However, previous studies showed that selfreports and hospital records were highly consistent for vascular disease [5, 33]. Lastly, lack of information on some other vascular risk factors (i.e., air pollution, depression, and sleep disorder) may cause inaccuracy in risk estimates based on the number of these risk factors.

\section{Conclusions}

Vascular risk factor burden is associated with the course of COVID-19 infection; patients with an increasing number of vascular risk factors have an increasingly high risk of severe COVID-19 disease and might benefit from specific COVID-19 prevention (e.g., self-isolation) and early hospital treatment measures. A better understanding of the pathophysiological mechanisms causing severe COVID-19 disease is needed to develop new treatment strategies for improving outcomes and reducing mortality in this high-risk population.

\section{Supplementary information}

Supplementary information accompanies this paper at https://doi.org/10. 1186/s12931-020-01510-0.

Additional file 1: Table S1. Association between vascular risk factor burden and severe COVID-19 illness adjusted for comorbidity, and sensitivity analyses using the E-value approach. Table S2. Association between vascular risk factor burden and severe COVID-19 illness adjusted for laboratory and chest CT findings, and sensitivity analyses using the Evalue approach. Figure S1. Bias Plots

\section{Abbreviations}

COVID: Coronavirus disease 2019; WHO: World Health Organization:

SD: Standard deviation; COPD: Chronic obstructive pulmonary disease;
IQR: Interquartile range; ALT: Alanine transaminase; AST: Alanine aminotransferase; LDH: Lactate dehydrogenase; CT: Computed tomography; HR: Hazard ratio

\section{Acknowledgments}

We thank all the patients with their data for this analysis and the medical workers who are on the front line of caring for patients. We thank Dr. Liming Lin (Xiamen University) for his assistance in statistical analysis.

\section{Authors' contributions}

Drs H Du and N Liu had full access to all of the data in the study and take responsibility for the integrity of the data and the accuracy of the data analysis. Drs H Du, N Liu, X Pan, J Chen and X Chen contributed equally to this work. Study concept and design: Drs H. Du, N. Liu, and D.J.Werring. Acquisition, analysis, or interpretation of data: Drs H Du, N Liu, X Pan, J Chen, X Chen, DJ Werring, G Ambler, X Li, Z Zheng, S Wu, H Lei, P Xia, Lei Gao, K Lin, M Huang, X Xu, C Chen, Y Luo, Z Zhao, C Li, R Cao, Yu Lin. Drafting of the manuscript: Drs H Du, N Liu, X Pan, J Chen, X Chen. Critical revision of the manuscript for important intellectual content: Drs. R Chen, Y Zhang, $H$ Huang, F Lin, C Li, H Lin, Z Huang, G Amber, D.J.Werring and L Chen. The authors read and approved the final manuscript.

\section{Funding}

This study was supported by the Fujian Provincial Special Foundation for Natural Science Innovation Project (2016B014) and the Fujian Science and Technology Innovation Joint Fund Project (2019Y9099). The funders had no role in the study design and the collection, analysis, and interpretation of data or drafting of the article and the decision to submit it for publication. The researchers confirm their independence from the funder.

\section{Availability of data and materials}

The datasets used and/or analyzed during the current study are available from the corresponding author on reasonable request (Email: houweidu@fjmu.edu.cn).

\section{Ethics approval and consent to participate}

The ethics committee of Fujian Medical University Union Hospital approved the study protocol (NO.2020GFKY005). All clinical investigations were conducted according to the principles expressed in the declaration of Helsinki. Written informed consent was waived due to the nature of our retrospective study of routine clinical data.

\section{Consent for publication}

Not applicable.

\section{Competing interests}

None.

\section{Author details}

'Stroke Research Center, Department of Neurology, Fujian Medical University Union Hospital, 29 Xinquan Road, Gulou District, Fuzhou 350001, China. ${ }^{2}$ Institute of Clinical Neurology, Fujian Medical University, Fuzhou, China. ${ }^{3}$ Department of Critical Care Medicine, Fujian Provincial Hospital South Branch, Fuzhou, China. ${ }^{4}$ Department of Rehabilitation, Fujian Medical University Union Hospital, Fuzhou, China. ${ }^{5}$ Department of Critical Care Medicine, Fujian Medical University Union Hospital, Fuzhou, China.

${ }^{6}$ Department of Infectious Disease, Fujian Medical University Union Hospital, Fuzhou, China. ${ }^{7}$ UCL Queen Square Institute of Neurology, London, UK. ${ }^{8}$ Statistical Science, University College London, London, UK. ${ }^{9}$ Fujian Center for Disease Control and Prevention, Fuzhou, China. ${ }^{10}$ Department of Neurology, Fuzhou Second Hospital Affiliated to Xiamen University, Fuzhou, China. ${ }^{11}$ Department of Thoracic Surgery, Fujian Medical University Union Hospital, Fuzhou, China. ${ }^{12}$ Department of Emergency Medicine, Fujian Medical University Union Hospital, Fuzhou, China. ${ }^{13}$ Department of Anesthesiology, Fujian Medical University Union Hospital, Fuzhou, China. ${ }^{14}$ Department of Cardiology, Fujian Medical University Union Hospital, Fuzhou, China. ${ }^{15}$ Department of Otolaryngology, Fujian Medical University Union Hospital, Fuzhou, China. ${ }^{16}$ Department of Radiology, Fujian Medical University Union Hospital, Fuzhou, China. ${ }^{17}$ Department of Colorectal Surgery, Fujian Medical University Union Hospital, Fuzhou, China. 
${ }^{18}$ Department of Respiratory Medicine, Fujian Medical University Union Hospital, Fuzhou, China.

Received: 23 July 2020 Accepted: 14 September 2020 Published online: 21 September 2020

\section{References}

1. Chen N, Zhou M, Dong X, Qu J, Gong F, Han Y, et al. Epidemiological and clinical characteristics of 99 cases of 2019 novel coronavirus pneumonia in Wuhan, China: a descriptive study. Lancet. 2020;395:507-13.

2. Novel coronavirus (2019-nCov) advice for the public. World Health Organization. https://www.who.int/emergencies/diseases/novelcoronavirus-2019/advice-for-public.2020. Accessed 14 Sep 2020.

3. Zhou F, Yu T, Du R, Fan G, Liu Y, Liu Z, et al. Clinical course and risk factors for mortality of adult inpatients with covid-19 in Wuhan, China: a retrospective cohort study. Lancet. 2020;395:1054-62.

4. Wu Z, McGoogan JM. Characteristics of and important lessons from the coronavirus disease 2019 (COVID-19) outbreak in China: summary of a report of 72314 cases from the Chinese Center for Disease Control and Prevention. JAMA. 2020;323:1239-42.

5. Yan R, Li W, Yin L, Wang Y, Bo J, Investigators PU-C. Cardiovascular diseases and risk-factor burden in urban and rural communities in high-, middleand low-income regions of China: a large community-based epidemiological study. J Am Heart Assoc. 2017;6:e004445.

6. Yusuf S, Joseph P, Rangarajan S, Islam S, Mente A, Hystad P, et al. Modifiable risk factors, cardiovascular disease, and mortality in 155722 individuals from 21 high-income, middle-income, and low-income countries (PURE): a prospective cohort study. Lancet. 2020;395:795-808.

7. Boukhris M, Hillani A, Moroni F, Annabi MS, Addad F, Ribeiro MH, et al. Cardiovascular implications of the covid-19 pandemic: a global perspective. Can J Cardiol. 2020;36:1068-80.

8. World Health Organization. Clinical management of severe acute respiratory infection when novel coronavirus ( $\mathrm{nCoV}$ ) infection is suspected: interim guidance. 2020. Accessed 5 Mar 2020

9. Du HW, Chen JN, Pan XB, Chen XL, Zhang YX, Fang SF, et al. Prevalence and outcomes of re-positive nucleic acid tests in discharged COVID- 19 patients. Eur J Clin Microbiol. 2020. https://doi.org/10.1007/s10096-02004024-1.

10. Li G, Guo G, Wang W, Wang K, Wang H, Dong F, et al. Association of prehypertension and cardiovascular risk factor clustering in Inner Mongolia: a cross-sectional study. BMJ Open. 2017;7:e01534010.

11. Emdin CA, Anderson SG, Salimi-Khorshidi G, Woodward M, MacMahon S, Dwyer $T$, et al. Usual blood pressure, atrial fibrillation and vascular risk: evidence from 4.3 million adults. Int J Epidemiol. 2017;46:162-72.

12. Wang $W$, Jiang $B$, Sun $H, R u X$, Sun $D$, Wang $L$, et al. Prevalence, incidence, and mortality of stroke in China: results from a nationwide populationbased survey of 480687 adults. Circulation. 2017;135:759-71.

13. Strath SJ, Kaminsky LA, Ainsworth BE, Ekelund U, Freedson PS, Gary RA, et al. Guide to the assessment of physical activity: clinical and research applications: a scientific statement from the American Heart Association. Circulation. 2013;128:2259-79.

14. Wu S, Wu B, Liu M, Chen Z, Wang W, Anderson CS, et al. Stroke in China: advances and challenges in epidemiology, prevention, and management. Lancet Neurol. 2019;18:394-405.

15. Wu C, Chen X, Cai Y, Xia J, Zhou X, Xu S, et al. Risk factors associated with acute respiratory distress syndrome and death in patients with coronavirus disease 2019 pneumonia in Wuhan, China. JAMA Intern Med. 2020;180:934-43.

16. VanderWeele TJ, Ding P. Sensitivity analysis in observational research: introducing the e-value. Ann Intern Med. 2017;167:268-74.

17. Guan WJ, Ni ZY, Hu Y, Liang WH, Ou CQ, He JX, et al. Clinical characteristics of coronavirus disease 2019 in China. N Engl J Med. 2020;382:1708-20.

18. Porcheddu R, Serra C, Kelvin D, Kelvin N, Rubino S. Similarity in case fatality rates (cfr) of covid-19/sars-cov-2 in Italy and China. J Infect Dev Ctries. 2020; $14: 125-8$.

19. Imam Z, Odish F, Gill I, O'Connor D, Armstrong J, Vanood A, et al. Older age and comorbidity are independent mortality predictors in a large cohort of 1305 COVID-19 patients in Michigan, United States. J Intern Med. 2020. https://doi.org/10.1111/joim.13119.

20. Samuels JD. Obesity and severe COVID-19 disease: a strong association. Obesity. 2020. https://doi.org/10.1002/oby.22866.
21. Ryan DH, Ravussin E, Heymsfield S. Covid 19 and the patient with obesity the editors speak out. Obesity. 2020;28:847.

22. Cai Q, Chen F, Wang T, Luo F, Liu X, Wu Q, et al. Obesity and covid-19 severity in a designated hospital in Shenzhen, China. Diabetes Care. 2020;43: 1392-8.

23. Sever $\mathrm{P}$, Johnston SL. The renin-angiotensin system and sars-cov-2 infection: a role for the ace2 receptor? J Renin-Angiotensin-Aldosterone Syst. 2020. https://doi.org/10.1177/1470320320926911.

24. Aleksova A, Ferro F, Gagno G, Cappelletto C, Santon D, Rossi M, et al. COVID-19 and renin-angiotensin system inhibition: role of angiotensin converting enzyme 2 (ACE2) - is there any scientific evidence for controversy? J Intern Med. 2020. https://doi.org/10.1111/joim.13101.

25. Gao C, Cai Y, Zhang K, Zhou L, Zhang Y, Zhang X, et al. Association of hypertension and antihypertensive treatment with COVID-19 mortality: a retrospective observational study. Eur Heart J. 2020;41:2058-66.

26. Li J, Wang X, Chen J, Zhang H, Deng A. Association of renin-angiotensin system inhibitors with severity or risk of death in patients with hypertension hospitalized for coronavirus disease 2019 (covid-19) infection in Wuhan, China. JAMA Cardiol. 2020;5:825-30.

27. Streiff MB, Agnelli G, Connors JM, Crowther M, Eichinger S, Lopes R, et al. Guidance for the treatment of deep vein thrombosis and pulmonary embolism. J Thromb Thrombolysis. 2016;41:32-67.

28. Minet C, Lugosi M, Savoye PY, Menez C, Ruckly S, Bonadona A, et al. Pulmonary embolism in mechanically ventilated patients requiring computed tomography: prevalence, risk factors, and outcome. Crit Care Med. 2012;40:3202-8.

29. Poyiadji N, Cormier P, Patel PY, Hadied MO, Bhargava P, Khanna K, et al. Acute pulmonary embolism and covid-19. Radiology. 2020. https://doi.org/ 10.1148/radiol.2020201955

30. Grillet F, Behr J, Calame P, Aubry S, Delabrousse E. Acute pulmonary embolism associated with covid-19 pneumonia detected by pulmonary ct angiography. Radiology. 2020;296:E186-8.

31. Simonnet A, Chetboun M, Poissy J, et al. High prevalence of obesity in severe acute respiratory syndrome coronavirus-2 (SARS-CoV-2) requiring invasive mechanical ventilation. Obesity. 2020;28:1195-9.

32. Driggin E, Madhavan MV, Bikdeli B, Chuich T, Laracy J, Biondi-Zoccai G, et al. Cardiovascular considerations for patients, health care workers, and health systems during the covid-19 pandemic. J Am Coll Cardiol. 2020;75:2352-71.

33. Yamagishi K, Ikeda A, Iso H, Inoue M, Tsugane S, Group JS. Self-reported stroke and myocardial infarction had adequate sensitivity in a populationbased prospective study jphc (Japan public health center)-based prospective study. J Clin Epidemiol. 2009;62:667-73.

\section{Publisher's Note}

Springer Nature remains neutral with regard to jurisdictional claims in published maps and institutional affiliations.
Ready to submit your research? Choose BMC and benefit from:

- fast, convenient online submission

- thorough peer review by experienced researchers in your field

- rapid publication on acceptance

- support for research data, including large and complex data types

- gold Open Access which fosters wider collaboration and increased citations

- maximum visibility for your research: over $100 \mathrm{M}$ website views per year

At $\mathrm{BMC}$, research is always in progress.

Learn more biomedcentral.com/submissions 